\title{
The investigation of evolution of the harmonic perturbation the stationary solution of the boundary value problem for a system of the Carleman equations
}

\author{
Olga Vasil'eva ${ }^{1, *}$ \\ ${ }^{1}$ Moscow state university of civil engineering, Yaroslavskoye shosse, 26, Moscow, Russia, 129337
}

\begin{abstract}
The evolution of the harmonic perturbation of one component of the stationary solution of the Carleman system is studied. The system can be used for mathematical modelling of technical problems in such fields: as the gas dynamics, the chemistry, acoustics etc. In particular, for the case of one dimensional space variable the system can be used to describe autokatalys problems for research of building materials. In this case the important property of the mathematical model is the stability of its stationary solutions. Numerical results of investigation of evolution perturbated stationary problem solution for special values of parameters are presented and analyzed
\end{abstract}

\section{Introduction}

We consider the evolution of the harmonic perturbation of the stationary solution of initial boundary problem for the kinetic Carleman system of equations. This system is a system of two nonlinear differential equation of first order. It can be used for mathematical modelling different problems of the kinetic theory of gasses, chemistry and in particular, for research of building materials. The Carleman system can be used to modelling autokatalys problem in the case one space variable. However the system has the main properties more complete system. It explains the fact that the investigation of the Carleman system is very interested now [1-6]. One of the important properties of the Carleman system is the stability of the stationary solutions. We consider the stationary solutions of the initial boundary problem for the Carleman system equation which components have opposite signs. The positive component of stationary solution is perturbated. The response of the solution to periodic perturbation is investigated. The investigation of the stationary solution properties is based on using the finite-difference methods and the application of quadrature formulas of the second order of accuracy

* Corresponding author: vasilijeva.ovas@yandex.ru 


\section{Formulation of the problem}

We consider the initial boundary problem for the Carleman system equations

$$
\begin{gathered}
u_{t}+u_{x}=\varepsilon^{-1}\left(w^{2}-u^{2}\right), \quad t>0,0<x<1 \\
w_{t}-w_{x}=\varepsilon^{-1}\left(u^{2}-w^{2}\right),
\end{gathered}
$$

with initial and boundary conditions

$$
\begin{gathered}
\left.u\right|_{t=0}=u^{0}(x),\left.w\right|_{t=0}=w^{0}(x) \\
u(t, 0)=u_{0}(t), w(t, 1)=w_{1}(t), u_{0}(0)=u^{0}(0), w_{1}(0)=w^{0}(1) .
\end{gathered}
$$

The functions $u^{\mathrm{s}}(t, x)=u^{\mathrm{s}}(x)=c$ and $w^{\mathrm{s}}(t, x)=w^{\mathrm{s}}(x)=-c$ are two components of the stationary solution (1) with following initial and boundary conditions $u^{0}(x)=c, w^{0}(x)=-c$, $u_{0}(t)=c, w_{1}(t)=-c$ for any constants $\varepsilon>0$ and $c>0$.

For the investigation properties of the stationary solutions we consider problem (1) with pertrubated initial condition (witn perturbated of the first pozitive component)

$$
u^{0}(x)=c-\alpha \sin (2 \pi \omega x), w^{0}(x)=-c
$$

and the following boundary conditions

$$
u_{0}(t)=c, w_{0}(t)=-c .
$$

The problem have parameters $\varepsilon, \alpha, c, \omega$.

Two components of energy of stationary solution perturbation $u^{\mathrm{s}}(x), w^{\mathrm{s}}(x)$ denote by

$$
\begin{gathered}
e_{1}(t)=\left(\int_{0}^{1}\left(u(t, x)-u^{s}(x)\right)^{2} d x\right)^{\frac{1}{2}} \\
e_{2}(t)=\left(\int_{0}^{1}\left(w(t, x)-w^{s}(x)\right)^{2} d x\right)^{\frac{1}{2}}
\end{gathered}
$$

and total enargy of perturbation denote by

$$
E(t)=\left(e_{1}^{2}(t)+e_{2}^{2}(t)\right)^{0.5}
$$

The maximum deviation of the first component solution from stationary solution $u^{s}(x)=c$ denote by

$$
D u(t)=\max _{0 \leq x \leq 1}|u(t, x)-c| \ldots
$$

The maximum deviation of the second component solution from stationary solution $w^{s}(x)=$ - $c$ denote by

$$
D u(t)=\max _{0 \leq x \leq 1}|w(t, x)+c|
$$

\section{Results of numerical investigation}

We consider the problem (1) - (3) for the $c=1.8, \varepsilon=0.57, \alpha=0.01, \omega=1$. Figures 1,2 present initial condition of problem $u^{0}(x), w^{0}(x)$. Figure 3, 4 show numerical solution of problem $u(t, x)$ and $w(t, x)$ the values of the time variable $t_{1}=1.5, t_{2}=3, t_{3}=4.5, t_{4}=6, t_{5}=$ 7.5..Figures 5, 6 demostrate the dependences of the components energy. Figure 7 demonstrates the total energy $\mathrm{E}(t)$ which tends to zero. The dependences have the oscillating character. 


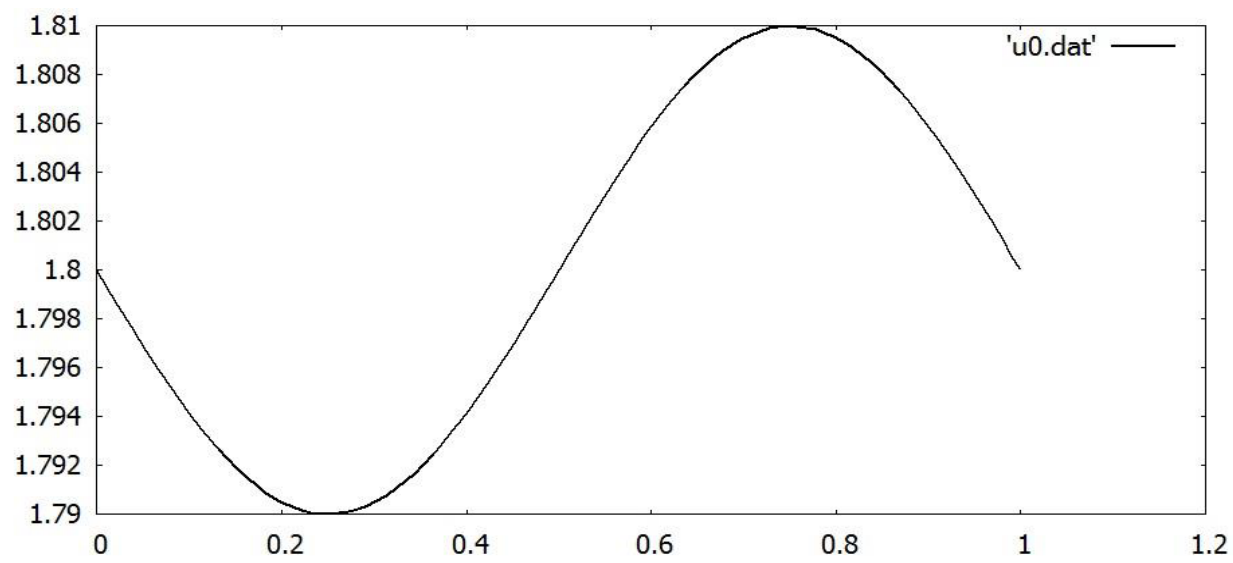

Fig. 1. Initial condition for the first solution component $u^{0}(x)$.

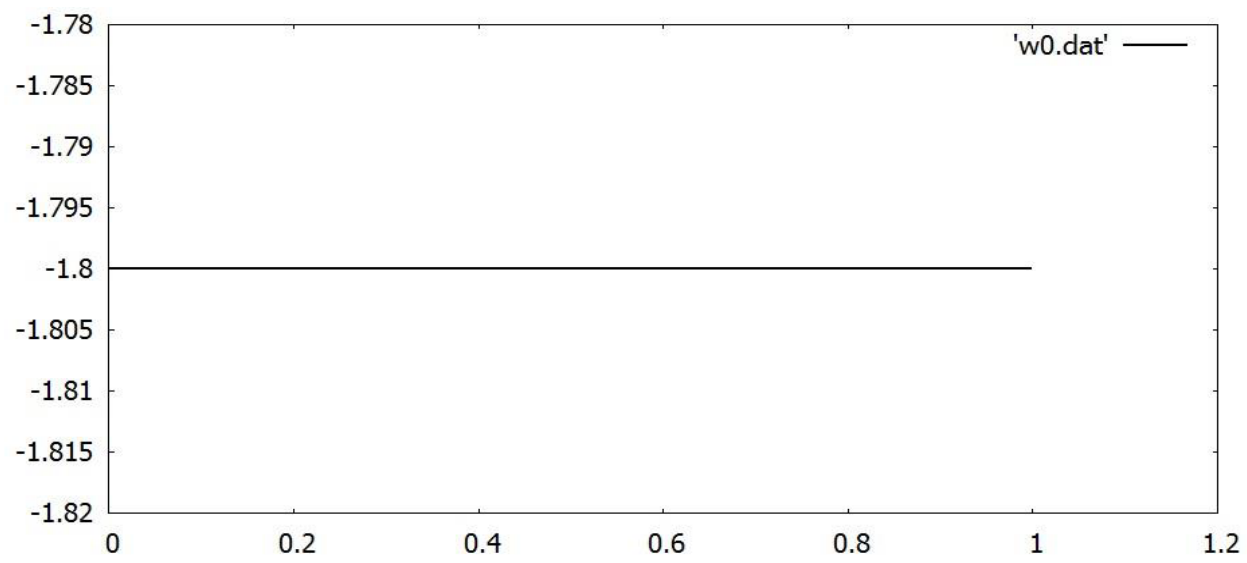

Fig.2. Initial condition for the second solution component $w^{0}(x)$.

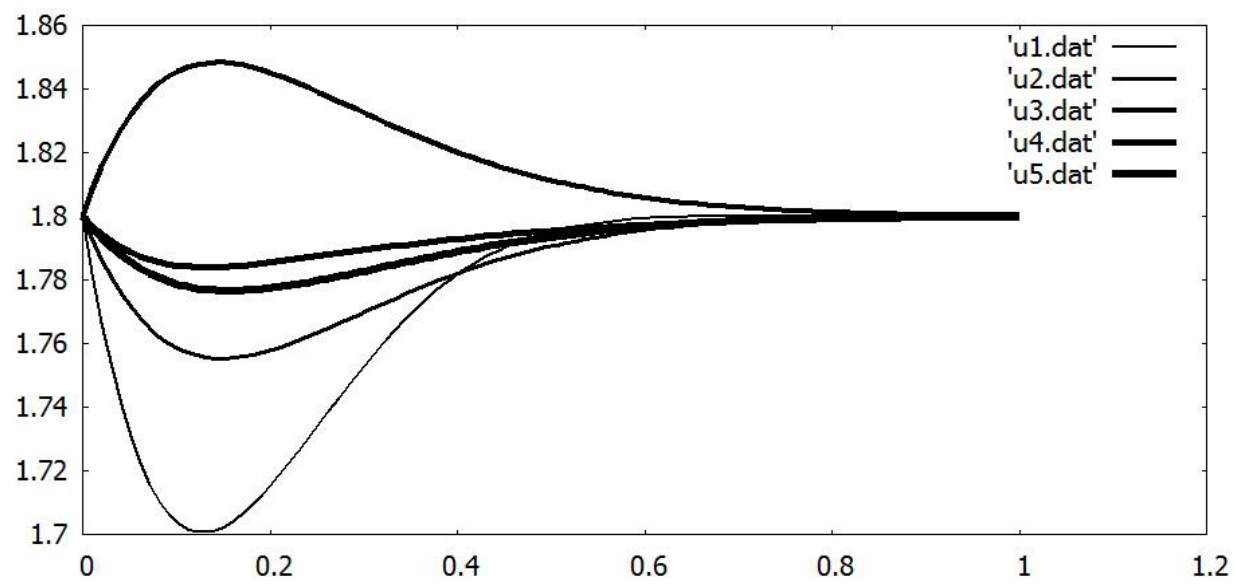

Fig. 3. The first component (the perturbated component) of numerical solution of problem $u(t, x)$. 


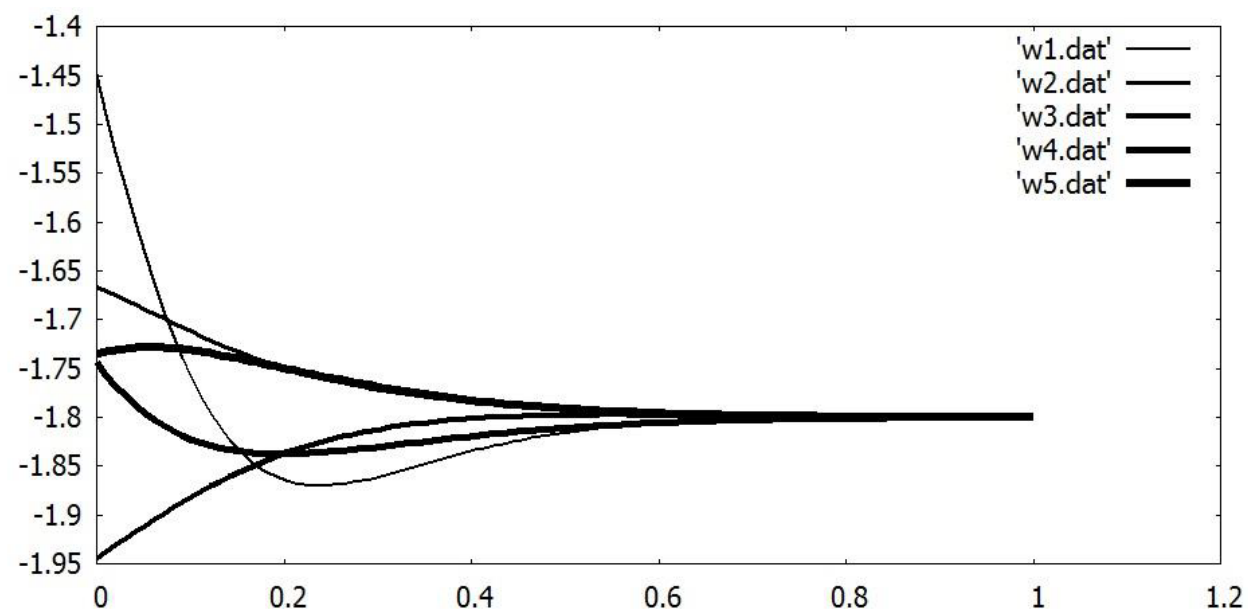

Fig. 4. . The second component of numerical solution of problem $w(t, x)$.

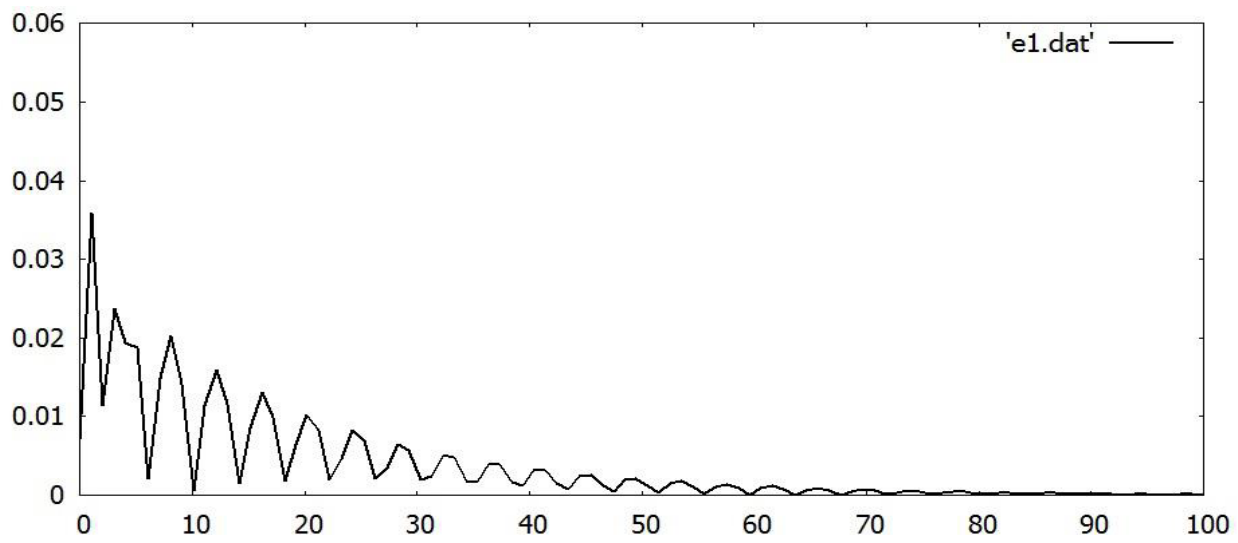

Fig. 5. Energy of perturbations $e_{1}(t)$.

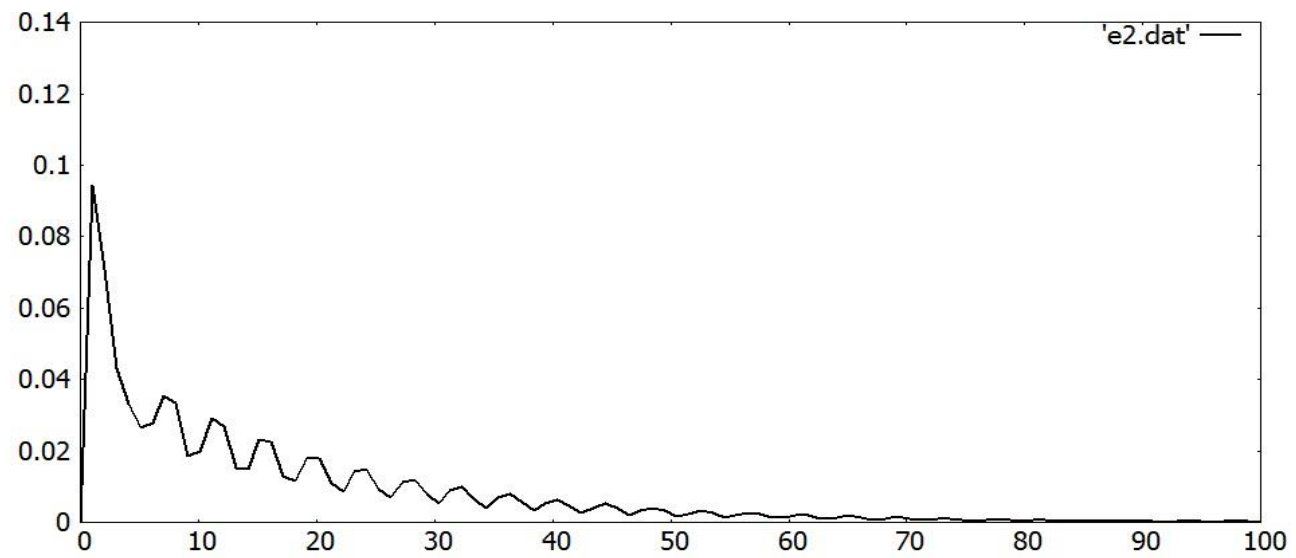

Fig. 6. Energy of perturbations $e_{2}(t)$. 


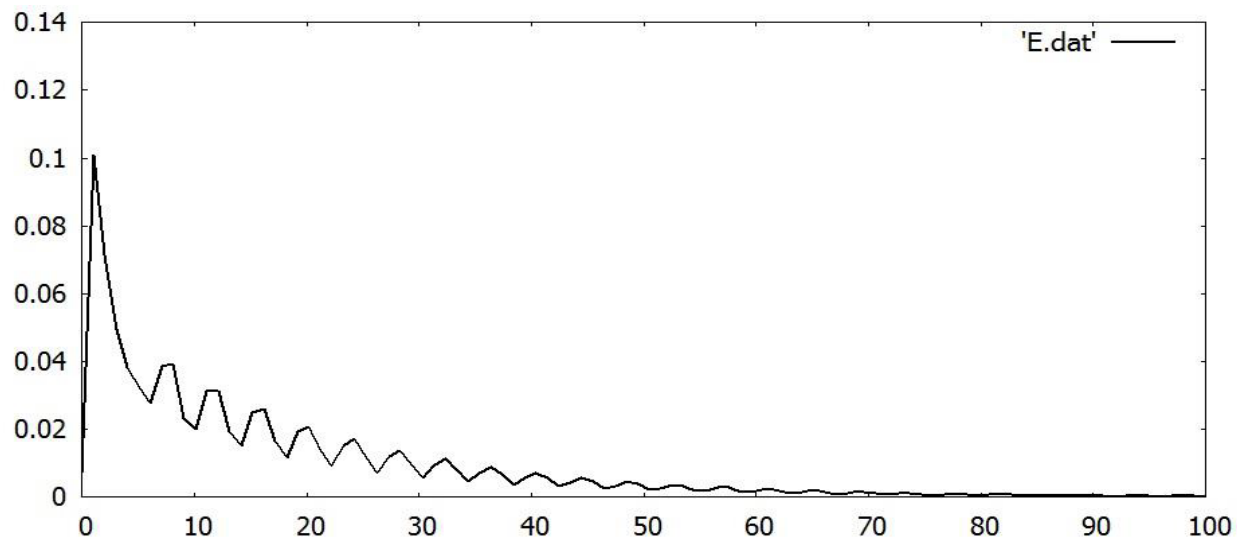

Fig. 7. Total energy $\mathrm{E}(t)$.

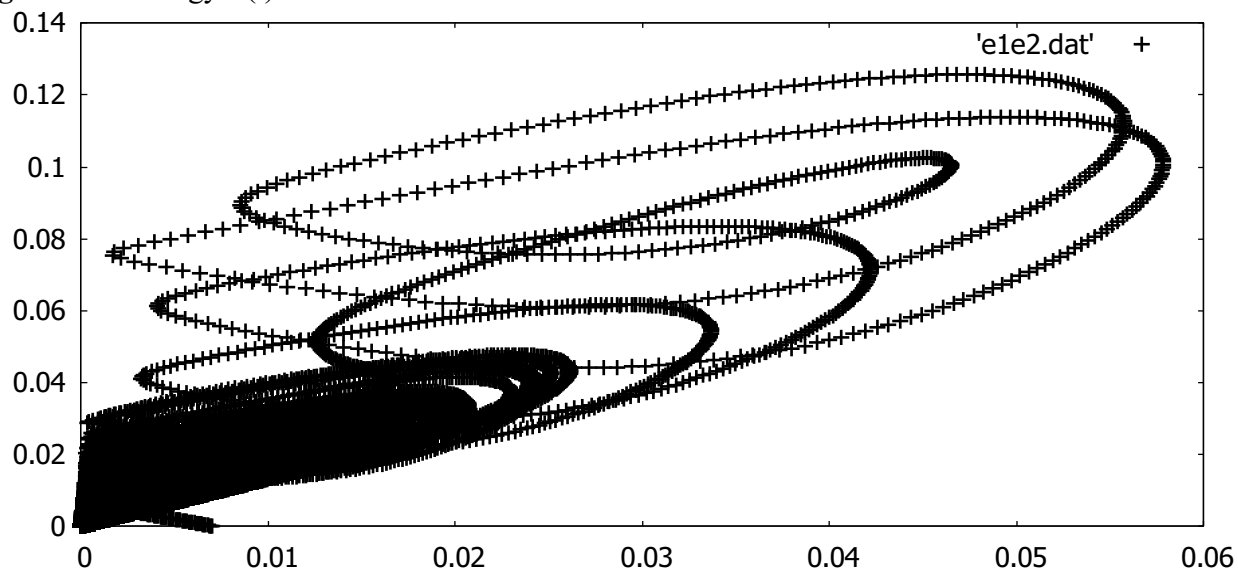

Fig. 8. Phase plane $\left(e_{1}(t), e_{2}(t)\right)$.

Figures 8 present the phase plane $\left(e_{1}(t), e_{2}(t)\right)$. Figure 9 demonstrates the dependences of maximum deviations of two component of the solution.

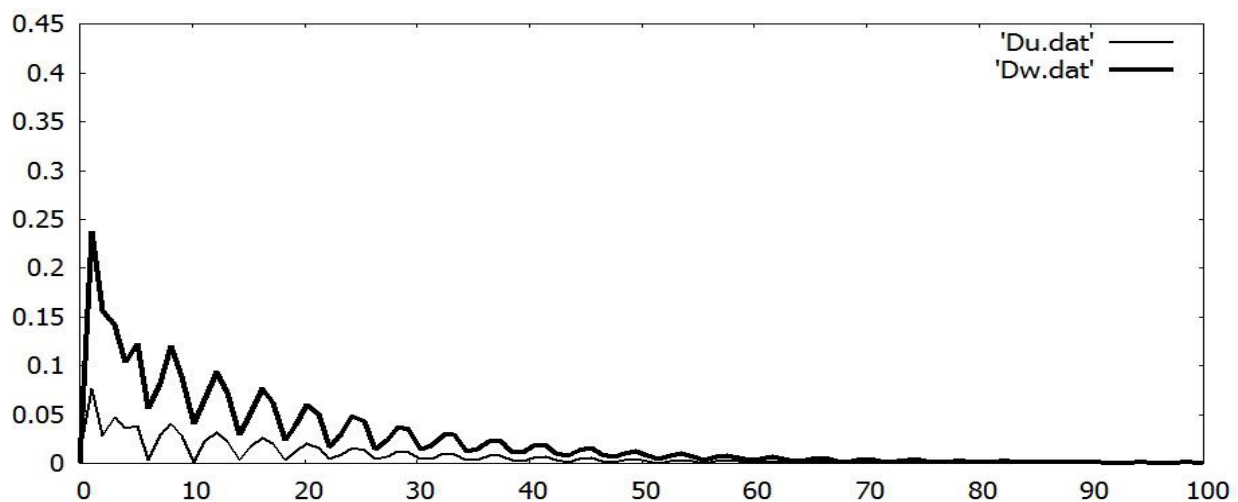

Fig. 9. Maximum deviations from stationary solution. 


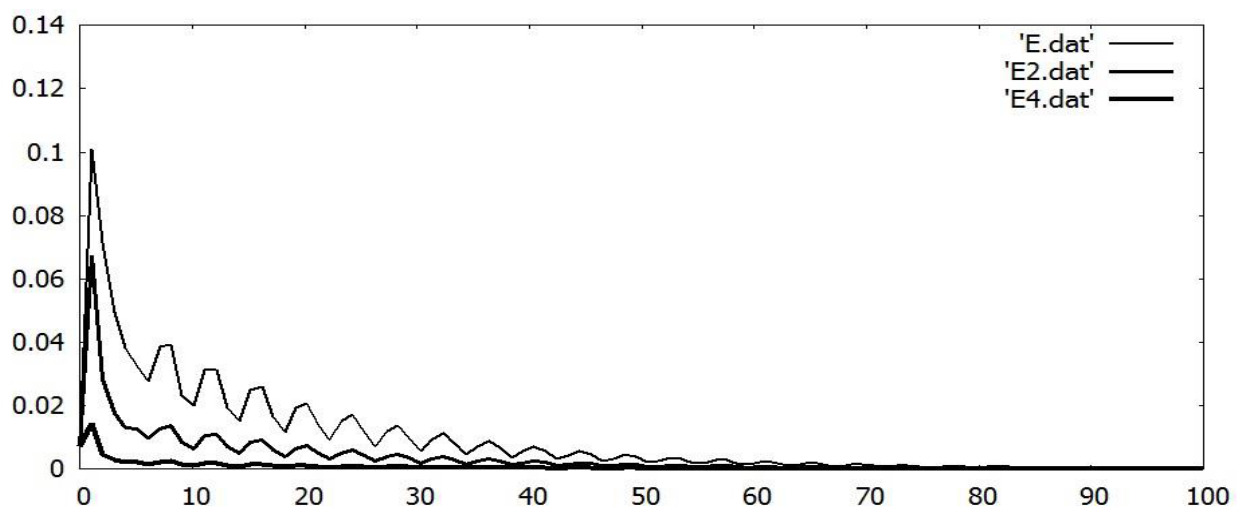

Fig. 10. Total energies for different $\omega$.

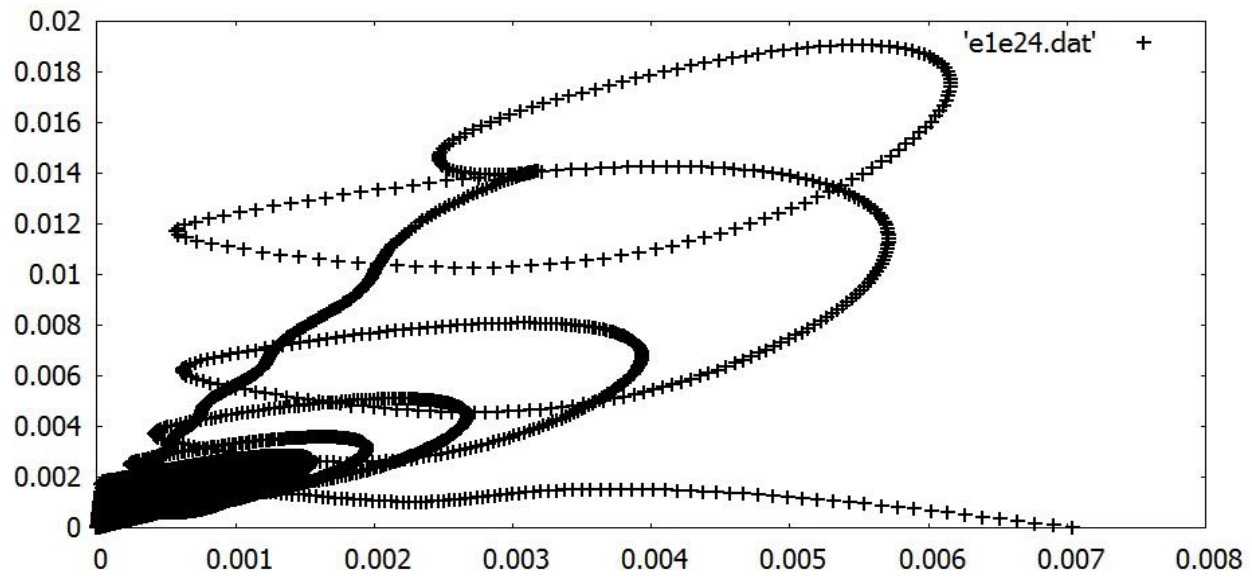

Fig. 11. Phase plane $\left(e_{1}(t), e_{2}(t)\right)$.

Figures 10 presents results of numerical investigation of the problem (1) - (3) for $c=-$ $1.8, \varepsilon=0.57, \alpha=0.01$ and different $\omega$. Curves E, E2, E4 correspond the following $\omega: 1,2,3$ Here we can see that the stabilisation time essentially increasing with growth $\omega$. But there is not the dependence between $\omega$ and the frequency of oscillation of the total energy $E(t)$. Figure 11 demonstrate the phase plane $\left(e_{1}, e_{2}\right)$ for $c=-1.8, \varepsilon=0.57, \alpha=0.01$ and $\omega=4$.

\section{Conclusion}

The results of numerical investigation of the perturbed stationary solution of the Carleman system for the case different sings of solution component are presented and analyzed. So, we can see that there is solution stabilization for considered values of parameters. Dependence of total energy and of maximal deviation solution from stationary solution has oscillating character with constant frequency. The stabilization time essentially depends from frequency perturbation of the first component initial condition. 
The author would like to express gratitude to Professor E.V.Radkevich for formulation of the problem and useful discussions and comments.

\section{References}

1. S. K. Godunov, U. M. Sultangazin. Math. Surv. 26, 3 (1971)

2. E. V. Radkevich. J. Math. Sci. 181, 5 (2012)

3. E. V. Radkevich. J. Math. Sci. 184, 4 (2012)

4. V. V. Aristov, O.V. Ilyin. Phys. Let. A. 374, 4381-4384 (2010)

5. E. V. Radkevich, O.A.Vasil'eva, S.A.Duhnovskij. J. Math. Sci. 201, 32 (2015)

6. E. V. Radkevich, O.A.Vasil'eva, J. Math. Sci. 224, 5 (2017) 